\title{
Efficacy of Botanical Pesticides against Sucking Insects Pests in Brinjal
}

\author{
S.K. Dehariya ${ }^{1 *}$, A. Shukla ${ }^{1}$, S.K. Barde ${ }^{1}$ and Kamlesh Ahirwar ${ }^{2}$ \\ ${ }^{1}$ Department of Entomology, JNKVV Jabalpur-482 004 (MP), India \\ ${ }^{2}$ Scientist Horticulture, Krishi Vigyan Kendra Chhatarpur (M.P.), India \\ *Corresponding author
}

\section{A B S T R A C T}

The experiment was conducted in randomized block design with 7 treatments and 4 replications, to evaluate the performance of some botanical products against the sucking

\section{Keywords}

Brinjal, Neem,

Botanicals, Jassids, Aphids

Article Info

Accepted:

14 December 2017

Available Online:

10 January 2018 pest of brinjal. The treatment included Triazophos 40E.C. $0.04 \%$, Neem oil $1 \%$, Achook 5 $\%$, NSKE 5\%, Karanj oil 1\%, Eucalyptus oil $1 \%$ and an untreated control. Four spraying of each treatment were conducted starting 30 days after transplanting, at an interval of 15 days. Observation on population of sucking pests (aphids and jassids) was recorded before treatment and 3, 7 and 10 days after each spraying. The results revealed that Triazophos 40E.C. $0.04 \%$ was significantly superior over all the botanical treatments. did not significantly shoot damage in different treatments ranged between 3.9 to $10.1 \%$. Highest healthy fruits yield $(24.76 \mathrm{q} / \mathrm{ha})$ was recorded in the treatment of Triazophos 40E.C. $0.04 \%$ followed by the treatment of neem oil $1 \%$ (20.54 q/ha healthy fruits), and both the treatments were statistically at par Yields in remaining treatments were at par and ranged between 19.57 and $15.23 \mathrm{q} / \mathrm{ha}$. Lowest yield (10.50 q/ha healthy fruits) was registered in untreated control. Highest cost benefit ratio of 1:6. 31 were in treatment of Triazophos 40 EC $0.04 \%$. Application of neem oil $1 \%$ registered the cost benefit ratio of 1:1.79 and found was most economical.

\section{Introduction}

Brinjal (Solanum melongena Linn.) is an important vegetable crop, in almost all parts of our country. The crop is generally sown twice or thrice in a year, depending upon the irrigation facilities. Many insect pests damage and affect the yield of brinjal crop to a great extent. Singh et al., (1984) have listed about 25 insect pests of brinjal, of which some major insect pests are brinjal shoot and fruit borer (Leucinodes orbonalis Gu.), Epilachna beetle (Epilachna vigintioctopunctata F.), aphids
(Aphis gossypi Glower), stem borer (Euzophera perticella Rag.) and jassid (Amrasca biguttula). Sucking insects pests are the serious pest of brinjal. The losses caused by various pests were estimated to be ranging from 28-85\% (Ahmed, 1974).

Suitable insecticides used for the control of brinjal pests are the important. However these insecticides cause the problem of resistance and resurgence of pests (Mehrotra, 1990). These toxic insecticides pollute environment and also adversely affect the natural enemies 
of pests. Several non-chemical means of pest management have been proposed for brinjal and other crops, like the manipulation of cultural practices, nutrient management, use of biological agents, etc.

To reduce pesticide hazards, one of the resorts is the application of insecticides of plant origin which are cheaper, easily available and safer to mankind. Neem plant has proved itself as a wonderful insecticide of plant origin, which is harmless to higher animals including man (Walunj et al., 1996). Plant products like Eucalyptus, Calotropis, Pongamia, Annona and Neem have been found effective in controlling for brinjal pests in green house.

Hence in present experiment meat the botanical product were tested. Tabaci is another serious and Polyphagous sucking pest of tomato crop. The damage is caused by both nymphs and adults. They suck the plant sap from lower Surface of leaves. Severe infestation results in premature defoliation and plant unable to produce flowers and fruit

\section{Materials and Methods}

The experiment was conducted to evaluate efficacy of botanical products against the major insect pest complex of brinjal at Vegetable Research Farm of College of Agriculture, JNKVV Jabalpur. The brinjal crop (Variety Pusa Purple Round) was raised by transplanting 26 days old seedlings in $4 \times 4$ meter plots with plant to plant and row to row distances of $45 \times 60 \mathrm{~cm}$. The crop was transplanted in the third week of April. Normal horticultural practices were followed to raise the crop. Experiment was planned with seven treatments and four replications following Randomized Block Design (RBD). Spacing of rows and plants distance was kept $60 \times 45 \mathrm{~cm}$. Four sprayings were done starting from 30 days after transplanting at an interval of 15 days. The spraying solution was taken @ of 500 liters water / ha. and spray using hand compression sprayer. Due care was exercised to eliminate the drift of spray material from one plot to.

Population of sucking pests (aphids and jassids) was recorded before treatment and 3,7and 10 days after each spraying. Three leaves of a single plant (upper, middle and lower) were observed to record the sucking pests Five random plants were observed in each treatment and replication throughout the crop season. Data of sucking pests was analysed using statistical analysis of variance at $5 \%$ level of significance. Suitable transformations were adopted before analysis of variance.

\section{Results and Discussion}

\section{Jassids}

The results are depicted in Table 1. The basis of 10 days after fourth spray the overall jassid population, found significantly superior over untreated control (27.75 per 15 leaves). However, among the botanicals, neem oil $1 \%$ (13.25/15 leaves), found superior over all the botanicals which at par with karanj oil $1 \%$ (14.25) and NSKP $5 \%$ (14.5/15 leaves) proved superior to rest of the botanical treatments. However Triazophos 40\% EC found superior over all the treatments. The next better treatment was eukalyptus oil $1 \%$ (15.25/15 leaves) and it was followed by Achook neem $0.5 \%$ (17/15 leaves). Earlier workers also tested the plant products against jassids and found reduction in the pest population by two plant extracts neem and karanj derivatives also obtained population reduction of jassids in neem treated plots which also produced higher yield than other treatments. Some findings were reported by previous workers Dimetry et al., 1996, Sabillon and Bustamante (1995) Somsekhara, et al., 1997, Singh et al., 2006. 
Table.1 Evaluation of different botanical products against jassids in brinjal crop

\begin{tabular}{|c|c|c|c|c|c|c|c|c|c|c|c|c|c|c|c|}
\hline \multirow{3}{*}{$\begin{array}{l}\text { S. } \\
\text { No. }\end{array}$} & \multirow[t]{3}{*}{ Treatments } & \multicolumn{14}{|c|}{ Mean jassids population per 15 leaves, day after } \\
\hline & & \multirow[t]{2}{*}{ Dose } & \multirow{2}{*}{$\begin{array}{c}\text { pre- } \\
\text { treatmen } \\
\mathrm{t}\end{array}$} & \multicolumn{3}{|c|}{ First spray } & \multicolumn{3}{|c|}{ Second spray } & \multicolumn{3}{|c|}{ Third spray } & \multicolumn{3}{|c|}{ Fourth spray } \\
\hline & & & & 3 days & 7 days & 10 days & 3 days & 7 days & 10 days & 3 days & 7 days & 10 days & 3 days & 7 days & 10 days \\
\hline 1. & $\begin{array}{c}\text { Triazophos } \\
40 \text { EC } 0.04 \%\end{array}$ & $1 \mathrm{ml} / \mathrm{lit}$. & $\begin{array}{c}23.50 \\
(4.819)\end{array}$ & $\begin{array}{c}9.00 \\
(2.988)\end{array}$ & $\begin{array}{c}13.00 \\
(3.599)\end{array}$ & $\begin{array}{c}15.00 \\
(3.859)\end{array}$ & $\begin{array}{c}6.75 \\
(2.593)\end{array}$ & $\begin{array}{c}11.00 \\
(3.308)\end{array}$ & $\begin{array}{c}16.00 \\
(3.982)\end{array}$ & $\begin{array}{c}8.00 \\
(2.789)\end{array}$ & $\begin{array}{c}8.00 \\
(2.814)\end{array}$ & $\begin{array}{c}14.00 \\
(3.736)\end{array}$ & $\begin{array}{c}3.00(1 \\
720)\end{array}$ & $\begin{array}{c}7.00 \\
(2642)\end{array}$ & $\begin{array}{c}9.00 \\
(2998)\end{array}$ \\
\hline 2. & Neem oil $1 \%$ & $10 \mathrm{ml} / \mathrm{lit}$. & $\begin{array}{c}22.50 \\
(4.742)\end{array}$ & $\begin{array}{c}11.00 \\
(3.294)\end{array}$ & $\begin{array}{c}12.00 \\
(3.449)\end{array}$ & $\begin{array}{c}18.75 \\
(3.859)\end{array}$ & $\begin{array}{c}7.25 \\
(4.327)\end{array}$ & $\begin{array}{c}7.25 \\
(2.669)\end{array}$ & $\begin{array}{c}17.00 \\
(4.109)\end{array}$ & $\begin{array}{c}15.00 \\
(3.885)\end{array}$ & $\begin{array}{c}7.00 \\
(2.628)\end{array}$ & $\begin{array}{c}10.00 \\
(3.152)\end{array}$ & $\begin{array}{c}16.00 \\
(2.428)\end{array}$ & $\begin{array}{c}9.00 \\
(2.998)\end{array}$ & $\begin{array}{c}13.25 \\
(3.634)\end{array}$ \\
\hline 3. & Achook 05\% & $5 \mathrm{ml} / \mathrm{lit}$. & $\begin{array}{l}21.75^{*} \\
(4.663)\end{array}$ & $\begin{array}{c}13.00 \\
(3.599)\end{array}$ & $\begin{array}{c}16.00 \\
(3.971)\end{array}$ & $\begin{array}{c}16.75 \\
(4.089)\end{array}$ & $\begin{array}{c}11.00 \\
(3.304)\end{array}$ & $\begin{array}{c}17.00 \\
(4.093)\end{array}$ & $\begin{array}{c}15.25 \\
(3.881)\end{array}$ & $\begin{array}{c}1100 \\
(3.308)\end{array}$ & $\begin{array}{c}12.50 \\
(3.532)\end{array}$ & $\begin{array}{c}17.00 \\
(4.119)\end{array}$ & $\begin{array}{c}8.00 \\
(2.826)\end{array}$ & $\begin{array}{c}13.00 \\
(3.599)\end{array}$ & $\begin{array}{c}17.00 \\
(4.119)\end{array}$ \\
\hline 4. & N.S.K.E $5 \%$ & $50 \mathrm{gm} / \mathrm{lit}$. & $\begin{array}{c}26.75 \\
(5.121)\end{array}$ & $\begin{array}{c}21.00 \\
(4.569)\end{array}$ & $\begin{array}{c}18.75 \\
(4.325)\end{array}$ & $\begin{array}{c}17.00 \\
(4.122)\end{array}$ & $\begin{array}{c}9.00 \\
(2.988)\end{array}$ & $\begin{array}{c}12.00 \\
(3.453)\end{array}$ & $\begin{array}{c}18.00 \\
(4.239)\end{array}$ & $\begin{array}{c}9.00 \\
(2.998)\end{array}$ & $\begin{array}{c}1100 \\
(3.313)\end{array}$ & $\begin{array}{c}15.75 \\
(4.030)\end{array}$ & $\begin{array}{c}11.00 \\
(3.315)\end{array}$ & $\begin{array}{c}12.50 \\
(3.535)\end{array}$ & $\begin{array}{c}14.50 \\
(3.803)\end{array}$ \\
\hline 5. & $\begin{array}{c}\text { Karanj oil } \\
1 \%\end{array}$ & $10 \mathrm{ml} / \mathrm{lit}$. & $\begin{array}{c}23.75 \\
(4.873)\end{array}$ & $\begin{array}{c}12.00 \\
(3.444)\end{array}$ & $\begin{array}{c}17.00 \\
(4.108)\end{array}$ & $\begin{array}{c}18.00 \\
(4.231)\end{array}$ & $\begin{array}{c}15.00 \\
(3.868)\end{array}$ & $\begin{array}{c}15.50 \\
(3.934)\end{array}$ & $\begin{array}{c}15.00 \\
(3.868)\end{array}$ & $\begin{array}{c}15.00 \\
(3868)\end{array}$ & $\begin{array}{c}17.00 \\
(4.122)\end{array}$ & $\begin{array}{c}17.50 \\
(4.183)\end{array}$ & $\begin{array}{c}11.75 \\
(3.419)\end{array}$ & $\begin{array}{c}14.25 \\
(3.773)\end{array}$ & $\begin{array}{c}14.25 \\
(3.747)\end{array}$ \\
\hline 6. & $\begin{array}{c}\text { Eucalyptus } \\
\text { oil } 1 \%\end{array}$ & $10 \mathrm{ml} / \mathrm{lit}$. & $\begin{array}{c}20.25 \\
(4.495)\end{array}$ & $\begin{array}{c}10.00 \\
(3.142)\end{array}$ & $\begin{array}{c}17.00 \\
(4111)\end{array}$ & $\begin{array}{c}19.00 \\
(4.355)\end{array}$ & $\begin{array}{c}8.75 \\
(2.948)\end{array}$ & $\begin{array}{c}13.00 \\
(3.597)\end{array}$ & $\begin{array}{c}13.25 \\
(3.633)\end{array}$ & $\begin{array}{c}9.00 \\
(2.985)\end{array}$ & $\begin{array}{l}17.00 \\
(419)\end{array}$ & $\begin{array}{c}17.75 \\
(4.213)\end{array}$ & $\begin{array}{c}11.00 \\
(3.315)\end{array}$ & $\begin{array}{c}14.50 \\
(3.749)\end{array}$ & $\begin{array}{c}15.25 \\
(3.880)\end{array}$ \\
\hline 7. & Untreated & - & $\begin{array}{c}25.00 \\
(4.999)\end{array}$ & $\begin{array}{c}27.00 \\
(5.196)\end{array}$ & $\begin{array}{c}27.00 \\
(5.190)\end{array}$ & $\begin{array}{c}29.00 \\
(5.385)\end{array}$ & $\begin{array}{c}29.00 \\
(5.385)\end{array}$ & $\begin{array}{c}27.75 \\
(5.266)\end{array}$ & $\begin{array}{c}29.25 \\
(5.407)\end{array}$ & $\begin{array}{c}25.75 \\
(5.024)\end{array}$ & $\begin{array}{c}28.00 \\
(5.291)\end{array}$ & $\begin{array}{c}28.00 \\
(5.291)\end{array}$ & $\begin{array}{c}24.50 \\
(4.898)\end{array}$ & $\begin{array}{c}29.00 \\
(5.194)\end{array}$ & $\begin{array}{c}27.75 \\
(5.243)\end{array}$ \\
\hline & CD $5 \%$ & & 0.238 & 0.546 & 0.456 & 0.352 & 0.427 & 0.500 & 0.541 & 0.701 & 0.339 & 0.302 & 0.344 & 0.374 & 0.453 \\
\hline & SEm \pm & & 0.006 & 0.033 & 0.023 & 0.014 & 0.020 & 0.028 & 0.033 & 0.055 & 0.013 & 0.010 & 0.013 & 0.015 & 0.023 \\
\hline
\end{tabular}

*Square root transformation 
Table.2 Evaluation of different botanical products against aphids in brinjal crop

\begin{tabular}{|c|c|c|c|c|c|c|c|c|c|c|c|c|c|c|c|}
\hline \multirow{3}{*}{$\begin{array}{c}\text { S. } \\
\text { No. }\end{array}$} & \multirow{3}{*}{$\begin{array}{c}\text { Treatment } \\
\text { s }\end{array}$} & \multicolumn{14}{|c|}{ Mean aphids population per 15 leaves, day after } \\
\hline & & \multirow[t]{2}{*}{ Dose } & \multirow[t]{2}{*}{ pre-treatment } & \multicolumn{3}{|c|}{ First spray } & \multicolumn{3}{|c|}{ Second spray } & \multicolumn{3}{|c|}{ Third spray } & \multicolumn{3}{|c|}{ Fourth spray } \\
\hline & & & & 3 days & 7 days & 10 days & 3 days & 7 days & 10 days & 3 days & 7 days & 10 days & 3 days & 7 days & 10 days \\
\hline 1. & $\begin{array}{c}\text { Triazophos } \\
40 \mathrm{EC} \\
0.04 \%\end{array}$ & $1 \mathrm{ml} / \mathrm{lit}$. & $23.50(4.845)$ & $\begin{array}{c}7.00 \\
(2.628)\end{array}$ & $\begin{array}{c}11.00 \\
(3.308)\end{array}$ & $\begin{array}{c}15.25 \\
(3.904)\end{array}$ & $\begin{array}{c}6.00 \\
(2.438)\end{array}$ & $\begin{array}{c}10.00 \\
(3.160)\end{array}$ & $\begin{array}{c}10.25 \\
(2 . .201)\end{array}$ & $\begin{array}{c}4.00 \\
(1.992)\end{array}$ & $\begin{array}{c}7.00 \\
(2.628)\end{array}$ & $\begin{array}{c}7.00 \\
(2.628)\end{array}$ & $\begin{array}{c}4.00 \\
(1.958)\end{array}$ & $\begin{array}{c}6.00 \\
(2428)\end{array}$ & $\begin{array}{c}7.00 \\
(2.628)\end{array}$ \\
\hline 2. & $\begin{array}{c}\text { Neem oil } \\
1 \%\end{array}$ & $10 \mathrm{ml} / \mathrm{lit}$. & $\begin{array}{c}24.75 \\
(4.974)\end{array}$ & $\begin{array}{c}13.00 \\
(3.602)\end{array}$ & $\begin{array}{c}17.00 \\
(4.119)\end{array}$ & $\begin{array}{c}19.50 \\
(4.414)\end{array}$ & $\begin{array}{c}11.00 \\
(3.308)\end{array}$ & $\begin{array}{c}11.25 \\
(2.998)\end{array}$ & $\begin{array}{c}13.75 \\
(3.706)\end{array}$ & $\begin{array}{c}10.00 \\
(3.160)\end{array}$ & $\begin{array}{c}14.25 \\
(3.770)\end{array}$ & $\begin{array}{c}17.25 \\
(4.149)\end{array}$ & $\begin{array}{c}7.50 \\
(2.731)\end{array}$ & $\begin{array}{c}10.00 \\
(3.152)\end{array}$ & $\begin{array}{c}13.00 \\
(3.604)\end{array}$ \\
\hline 3. & $\begin{array}{c}\text { Achook } \\
05 \%\end{array}$ & $5 \mathrm{ml} / \mathrm{lit}$. & $\begin{array}{c}26.00 \\
(5.147)\end{array}$ & $\begin{array}{c}12.00 \\
(3.457)\end{array}$ & $\begin{array}{c}15.50 \\
(3.931)\end{array}$ & $\begin{array}{c}21.00 \\
(4.580)\end{array}$ & $\begin{array}{c}12.00 \\
(3.463)\end{array}$ & $\begin{array}{c}13.00 \\
(3.599)\end{array}$ & $\begin{array}{c}19.00 \\
(4.358)\end{array}$ & $\begin{array}{c}14.00 \\
(3.740)\end{array}$ & $\begin{array}{c}19.00 \\
(4.358)\end{array}$ & $\begin{array}{c}23.00 \\
(4.793)\end{array}$ & $\begin{array}{c}9.75 \\
(3.114)\end{array}$ & $\begin{array}{c}13.00 \\
(3.599)\end{array}$ & $\begin{array}{c}21.25 \\
(4.605)\end{array}$ \\
\hline 4. & $\begin{array}{c}\text { N.S.K.E } \\
5 \%\end{array}$ & $50 \mathrm{gm} / \mathrm{lit}$. & $\begin{array}{c}27.00 * \\
(5.94)\end{array}$ & $\begin{array}{c}13.75 \\
(3.703)\end{array}$ & $\begin{array}{c}12.00 \\
(3.461)\end{array}$ & $\begin{array}{c}24.00 \\
(4.896)\end{array}$ & $\begin{array}{c}17.00 \\
(4.119)\end{array}$ & $\begin{array}{c}19.00 \\
(4.358)\end{array}$ & $\begin{array}{c}21.00 \\
(4.582)\end{array}$ & $\begin{array}{c}12.00 \\
(3.463)\end{array}$ & $\begin{array}{c}13.50 \\
(3.737)\end{array}$ & $\begin{array}{c}16.00 \\
(3.995)\end{array}$ & $\begin{array}{c}9.00 \\
(2.988)\end{array}$ & $\begin{array}{c}16.00 \\
(3.995)\end{array}$ & $\begin{array}{c}16.00 \\
(4.056)\end{array}$ \\
\hline 5. & $\begin{array}{c}\text { Karanj oil } \\
1 \%\end{array}$ & $10 \mathrm{ml} / \mathrm{lit}$. & $24.5(4.948)$ & $\begin{array}{c}10.00 \\
(3.154)\end{array}$ & $\begin{array}{c}13.00 \\
(3.604)\end{array}$ & $\begin{array}{c}19.00 \\
(4.358)\end{array}$ & $\begin{array}{c}13.00 \\
(3.599)\end{array}$ & $\begin{array}{c}16.75 \\
(4.089)\end{array}$ & $\begin{array}{c}20.00 \\
(5.383)\end{array}$ & $\begin{array}{c}8.00 \\
(2.826)\end{array}$ & $\begin{array}{c}15.00 \\
(3.675)\end{array}$ & $\begin{array}{c}21.80 \\
(4.581)\end{array}$ & $\begin{array}{c}7.00 \\
(2.628)\end{array}$ & $\begin{array}{c}8.00 \\
(2.814)\end{array}$ & $\begin{array}{c}13.50 \\
(3.666)\end{array}$ \\
\hline 6. & $\begin{array}{c}\text { Eucalyptus } \\
\text { oil } 1 \%\end{array}$ & $10 \mathrm{ml} / \mathrm{lit}$. & $25.5(5.049)$ & $\begin{array}{c}15.75 \\
(4.062)\end{array}$ & $\begin{array}{c}18.75 \\
(4.327)\end{array}$ & $\begin{array}{c}20.50 \\
(4.526)\end{array}$ & $\begin{array}{c}13.00 \\
(3.604)\end{array}$ & $\begin{array}{c}17.00 \\
(4.119) .\end{array}$ & $\begin{array}{c}21.00 \\
(4.579)\end{array}$ & $\begin{array}{c}11.00 \\
(3.315)\end{array}$ & $\begin{array}{c}9.00 \\
(3.036)\end{array}$ & $\begin{array}{c}13.00 \\
(3.599)\end{array}$ & $\begin{array}{c}10.00 \\
(3.152)\end{array}$ & $\begin{array}{c}15.25 \\
(3.897)\end{array}$ & $\begin{array}{c}18.75 \\
(4.324)\end{array}$ \\
\hline 7. & Untreated & - & $28.00(5.289)$ & $\begin{array}{c}26.00 \\
(5.097)\end{array}$ & $\begin{array}{c}26.00 \\
(5.097)\end{array}$ & $\begin{array}{c}29.00 \\
(5.383)\end{array}$ & $\begin{array}{c}30.00 \\
(5.477)\end{array}$ & $\begin{array}{c}30.75 \\
(5.544)\end{array}$ & $\begin{array}{c}33.00 \\
(5.744)\end{array}$ & $\begin{array}{c}29.00 \\
(5.384)\end{array}$ & $\begin{array}{c}32.00 \\
(3.657)\end{array}$ & $\begin{array}{c}29.00 \\
(5.385)\end{array}$ & $\begin{array}{c}24.75 \\
(4.974)\end{array}$ & $\begin{array}{c}25.50 \\
(3.049)\end{array}$ & $\begin{array}{c}25.75 \\
(3.073)\end{array}$ \\
\hline & CD $5 \%$ & & 0.232 & 0.370 & 0.283 & 0.222 & 0.287 & 0.275 & 0.183 & 0.162 & 0.450 & 0.332 & 0.417 & 0.379 & 0.360 \\
\hline & SEm \pm & & 0.006 & 0.015 & 0.009 & 0.005 & 0.009 & 0.008 & 0.003 & 0.003 & 0.023 & 0.012 & 0.019 & 0.16 & 0.14 \\
\hline
\end{tabular}

Table.3 Economics of control operations by various botanical products

\begin{tabular}{|c|c|c|c|c|c|c|}
\hline S.No. & Treatments & $\begin{array}{l}\text { Yields of healthy } \\
\text { fruits (q/ha) }\end{array}$ & $\begin{array}{l}\text { Increase in yield } \\
\text { over untreated } \\
\text { control }\end{array}$ & $\begin{array}{l}\text { Value of } \\
\text { increased } \\
\text { yield }(\%)\end{array}$ & $\begin{array}{c}\text { Cost of treatment/ha (Rs.) } \\
\text { (labour + material cost for } 4 \\
\text { sprays) }(\%)\end{array}$ & $\begin{array}{c}\text { Cost benefit } \\
\text { ratio }\end{array}$ \\
\hline 1. & Triazophos 40 EC $0.04 \%$ & 24.76 & 14.26 & 9982 & 1580 & $1: 6.31$ \\
\hline 2. & Neem oil $1 \%$ & 20.54 & 10.04 & 7028 & 5960 & $1: 1.79$ \\
\hline 3. & Achook $0.5 \%$ & 15.23 & 4.73 & 3311 & 4700 & $1: 0.70$ \\
\hline 4. & N.S.K.E. $3 \%$ & 17.28 & 6.78 & 4746 & 12560 & $1: 0.37$ \\
\hline 5. & Karanj oil $1 \%$ & 17.81 & 7.31 & 5117 & 5360 & 1:0.95 \\
\hline 6. & Eucalyptus oil1\% & 19.57 & 9.07 & 6349 & 22160 & $1: 0.28$ \\
\hline 7. & Untreated control & 10.50 & - & 1050 & 7350 & \\
\hline
\end{tabular}




\section{Aphids}

The results are depicted in Table 2. All the treatments had less leaf infestation than untreated control (25.75 mean aphid population/15 leaves). Among all the treatments triazophos $40 \% \mathrm{EC}$ found superior over all the treatments (7.00 mean aphid population/15 leaves), among botanicals neem oil 1\% (13.00/15 leaves), found superior over all the botanicals which at par with karanj oil $1 \%$ (13.50) and NSKP $5 \%$ (16.00/15 leaves) proved significantly superior than other treatments. The next better treatment was Achook neem $0.5 \%$ (21.00/ 15 leaves). The above finding was found more or less similarly to the previous workers Trinidade et al., (2000) also reported 82 to 94.7 per cent mortality of eggs and larvae of tomato leaf miner fourth day after the treatment of neem seed kernel extract (NSKE).

However, 100 per cent mortality was caused by all the concentrations after sixth day of the treatment. Jayakumar and Uthamasamy (1997) also reported that neem oil 3\% and mahua oil 3\% caused 93.3 per cent and 90 per cent larval mortality of Myzus persicae. Viraktamath et al., (1993) also reported the effectiveness of neem seed kernel extract $4 \%$ against Liriomyza trifolii on tomato. Azam (1991) reported that the neem oil 1.0 and 1.25 per cent caused more than 80 per cent mortality of the larvae and pupae of $L$. trifolii and other similar findings were reported by Murthy and Prasad (1996) Wankhede et al., (2007) Mishra and Shantipriya (2008). The maximum healthy fruit yield was recorded in Triazophos 40\% EC (24.76 Quintal/ha) neem oil $1 \%(20.54 \mathrm{q} / \mathrm{ha})$ which was at par with eukalyptus oil $1 \% \quad(19.5 \mathrm{q} / \mathrm{ha})$ proved significantly superior to other treatments. The Minimum fruit yield was rerecorded in untreated control plot $(10.5 \mathrm{q} / \mathrm{ha})$ it is two times less than neem oil $1 \%$.

\section{References}

Ahmad, R. 1974. Studies on the pests of brinjal and their control with special reference to fruit borer, Leucinodes orbonalis Guen (Pyralidae: Lepidoptera). Entomologist's News Letter. 1(4): 2-3

Azam, K.M. 1991. Txicity of neem oil against leafminer (Liriomyza sativa. Burgass) on cucumber. Pl.Prot.Qtly. 6(4): 196197.

Dimetry, N.Z.; Gomaa, A.A., Salem, A.A. and Abd-El-Moniem, A.S.H. 1996. Bioactivity of some formulations of neem seed extracts against the white fly Bemisia tabacii (Genn.). Anzeiger-FurSchadlingshunde, - Pflanzenschutz, Umweltschutz. 69(6): 140-141.

Jayakumar, P. and Uthamasamy, S. 1997. Bio-efficacy of some synthetic insecticides and botanicals against Liriomyza trifolii Indian. J. Ent., 59(4): 347-350.

Mehrotra. K.N. 1990. Pyrethroids resistance in pest management. Indian Experience Pestic. Res. J., 2(1): 44-52.

Mishra, H.P. and Shantipriya, B. 2008. Mangement of serpentine leaf miner Liriomyza trifolii in okra using biopesticides. Indian J. Plant Prot. 36(1): 130-131.

Murthy, K.S. and Prasad, Y.G. 1996. Management of serpentine leaf miner on caster. Insect Environment. 2(1): 4-5.

Sabillon, A. and Bastamante, M. 1995. Evaluation of botanical extracts for the control of tomato pests (Lycopersicon esculentum Mill.). CETBA, 36(2): 179187.

Singh Swaroop, Choudhary, D.P. and Mathur, Y.S. 2006. Effect of plant extract against white fly (Bemisia tabaci Genn.) on tomato. Indian J. Entomology. 68(1): 71-73.

Singh, H. 1984. Household and kitchen 
garden pests, principles and practices, Kalyani Publications, Daryaganj, New Delhi. Pp. 168-169.

Somsekhara, Y.M., Nateshan,H.M. and Muniyappa, V. 1997. Evaluations of neem products and insecticides against white fly (Bemisia tabaci Genn.) a vector of tomato leaf curl gemini virus disease. Indian Journal Plant Prot. 25(1): 56-59.

Trinidade, R.C.P., I.M.R. Marques, H.S. Xavier, J.V. Oliveira and J.V. deOliveira, 2000. Neem seed kennel extract and the tomato leaf miner egg and larval mortality. Scientia - Agricola, 57(3): 407-419.

Vikraktamath, C.A., G.C. Tiwari, K.
Srinivasan and M. Gupta (1993). American serpentine leaf miner is a new threat to crops. Indian Fmg., 43: 10-12.

Walunj, A.R., U.N. Mote, AC. Desai and K.M. Parikh 1996. Efficacy of 29-199, a Neem based insecticide against brinjal shoot and fruit borer. Pestology. 20(1): 7-9.

Wankhede, S.M., Deotale, V.Y., Undirwade, D.B., Mane, P.N., Deotale, R.O. and Khare, R.N. 2007. Performance of some insecticides and bio-pesticides against tomato leaf miner (Liriomyza sativa. Burgass) J. Soils and Crops, 17: 136138.

\section{How to cite this article:}

Dehariya, S.K., A. Shukla, S.K. Barde and Kamlesh Ahirwar. 2018. Efficacy of Botanical Pesticides against Sucking Insects Pests in Brinjal. Int.J.Curr.Microbiol.App.Sci. 7(01): 19301935. doi: https://doi.org/10.20546/ijcmas.2018.701.232 